\title{
EL SISTEMA ELECTORAL ITALIANO Y LA INNOVACIÓN DEL ART. 122 DE LA CONSTITUCIÓN
}

\author{
FULCO LANCHESTER
}

Profesor Ordinario de Derecho Constitucional Universidad de Roma "La Sapienza" 
SUMARIO

1. Premisa. 2. Alternativa actual: la fase constituyente y la ley constituCIONAL DEL 22 DE NOVIEMBRE, NÚM. 1 - 1999. 3. AlgunAS PUNTUALIZACIONES CONCEPTUALES SOBRE MATERIA ELECTORAL. 4. DINÁMICA DE ESTA DISCUSIÓN EN LA NORMATIVA DE LA DEMOCRACIA PLURALISTA. 5. EL CASO ITALIANO Y SUS PECULIARIDADES. 6. LOS PRECEDENTES Y EL ITER DE LA INNOVACIÓN CONSTITUCIONAL DE 1999. 7. La INNOVACIÓN CONSTITUCIONAL DEL ART. 122. 8. CONCLUSIONES: ALTERNATIVAS POSIBLES Y PELIGROS DE UNA SITUACIÓN CONFUSA. 


\title{
EL SISTEMA ELECTORAL ITALIANO Y LA INNOVACIÓN DEL ART. 122 DE LA CONSTITUCIÓN *
}

POR

\author{
FULCO LANCHESTER \\ Profesor Ordinario de Derecho Constitucional \\ Universidad de Roma "La sapienza»
}

\section{PREMISA}

La ley constitucional del 22 de noviembre 1999, núm. 1 y, en especial, la innovación introducida por la misma en el art. 122 de la Constitución, ocupan el centro de esta breve relación. Dicho tema une el argumento sobre las formas de gobierno con el del sistema electoral en sentido estricto. En efecto, los dos temas sólo pueden separarse desde el punto de vista analítico, pero no desde el práctico. Ciertamente, el sistema electoral y la forma de gobierno constituyen un conjunto de instrumentos institucionales que, dentro del cuadro de la forma de Estado y del régimen en vigor, retroactúan y producen efectos diferenciados en relación con el panorama partidista y el significado que asume la discusión. En el caso de la forma de gobierno regional, la coherencia del modelo propuesto por la ley constitucional

Texto basado en una intervención del autor, previa al referendum de 21 de mayo de 2000, y a las elecciones parlamentarias de 2001 . 
en cuestión ( $\mathrm{y}$, en particular, cuanto está previsto por las normas transitorias) requiere una integración estrecha de los instrumentos institucionales referentes a la forma de gobierno $y$ al sistema electoral, habida cuenta de que este último -por encima de su posición formal dentro de la jerarquía de las fuentes- constituye un instrumento técnico de gran valor político con características de verdadera y auténtica norma de régimen que, incluso en la fase fundante, tiende a conectar con el panorama y los intereses de los sujetos que deciden las reglas.

Para mayor claridad, indico desde el principio cómo me moveré. Antes de entrar en materia, expondré algunas consideraciones generales necesarias sobre la fase institucional italiana. A continuación, presentaré varias distinciones conceptuales en el ámbito del sector, más amplio, de la legislación electoral, para después ofrecer la dinámica de los instrumentos adoptados en el tiempo a nivel comparativo para cuanto se refiere al tema general, tanto desde el punto de vista horizontal como vertical. Seguidamente afrontaré los aspectos problemáticos de la nueva normativa, para examinar luego las incógnitas de la transición institucional actual, sobre todo en la relación entre los sectores nacional y autonómico.

\section{ALTERNATIVA ACTUAL. LA FASE CONSTITUYENTE Y LA LEY CONSTITUCIONAL DEL 22 DE NOVIEMBRE, NÚM. 1 - 1999}

En el debate político de estos últimos meses, como también hace unos años, se ha hablado de verdadera y auténtica fase constituyente en lo referente al nivel regional. Desde el punto de vista hermenéutico resulta preliminar, en mi opinión, resolver este interrogante más general a fin de desarrollar adecuadamente el análisis más específico.

A este propósito, me resulta evidente que, desde el punto de vista técnico-jurídico, la definición de la fase que se ha abierto con la innovación constitucional como fase constituyente, no sólo no es correcta, sino que puede ser peligrosa por la potencialidad de conflicto y de frustraciones que podrían derivarse de ella. Porque la aceptación de la tesis constituyente con todas sus implicaciones lógicas, implica que las regiones ejerzan un poder libre en su fin y no condicionado por otros. Desde la óptica jurídica, en cambio, la ley constitucional en cuestión atribuye a las regiones competencias concretas e importantes en materias que antes regulaba directamente la Constitución y la ley estatal; pero la misma ley constitucional la delimita, directamente o renviándola a una futura ley estatal de principios. 
Resulta diáfano, entonces, que el adjetivo constituyente, en el ámbito de la argumentación en curso no posee un significado técnicojurídico preciso, sino más bien un valor semántico ambiguo que, entre otras cosas, identifica la posibilidad de las regiones de ensanchar de hecho los límites fijados a sus opciones en cuanto a la organización interna sobre la base de una situación fluida de la vida política nacional. Con tal perspectiva, entiendo que es necesario tener en cuenta -a fin de interpretar correctamente las normas en cuestión- la duplicidad de los planes programados y evitar mezclarlos; teniendo en cuenta por otro lado, los intereses normativos que resultan oscuros por la incapacidad de los legisladores para esclarecerlos y por la neblina con que se habla de la misma ley sobre los principios, que deberia ser aprobada por un Parlamento nacional en periodo preelectoral con Consejos regionales apenas elegidos.

En mi óptica, la fase que se abre ante las instituciones regionales no es, por tanto, constituyente sino reconstructiva dentro del complejo proceso de realineación del sistema partidista e institucional a distintos niveles. En esta dimensión específica, uno de los peligros reside en que también sobre la base de las incertidumbres constantes a nivel nacional, haya incoherentes diferencias respecto del modelo propuesto por la ley constitucional, partiendo de la variedad de panoramas partidistas de referencia; y también en que la incisiva reestructuración de las diferencias Junta-Consejo lleve a tensiones y alteraciones derivadas de la idiosincrasia de los sujetos implicados en la discusión de las reglas.

\section{ALGUNAS PUNTUALIZACIONES CONCEPTUALES SOBRE MATERIA ELECTORAL}

Dicho esto y como mera introducción y estímulo para el debate, para cuanto se refiere al tema electoral es oportuno recordar brevemente algunas definiciones que pueden proporcionar un marco conceptual para la discusión en acto:

a) por legislación electoral general se entiende el conjunto de normas enderezado al cumplimiento del acto electoral;

b) por capacidad electoral activa y pasiva se entiende la idoneidad (o no idoneidad) para participar como sujeto activo o pasivo en el debate electoral o causas que se lo impiden;

c) por sistema electoral en sentido estricto se entiende el mecanismo de transformar los votos en escaños, constituido por el 
tipo de opción, tipo y dimensión del colegio y tipo de fórmula (proporcional o mayoritario);

d) por legislación electoral de entorno se entiende toda la normativa consiguiente $y$, en particular, el ámbito de la propaganda y financiación del acto electoral. Desde el punto de vista conceptual, la legislación electoral está inserta en el ámbito de las votaciones públicas; y la línea de demarcación de la intervención pública constituye una decisión de vastísima relevancia para indicar el contexto procedimental del desenvolvimiento de la legislación del sector. Para mayor claridad, el área de intervención de cada sector individual puede ampliarse hasta la selección misma de los candidatos, cuando la normativa considere importante tal solución.

\section{LA DINÁMICA DE ESTA DISCUSIÓN EN LA NORMATIVA DE LA DEMOCRACIA PLURALISTA}

Circunscrito de este modo el campo de acción y no profundizando más por el momento, es oportuno tener en cuenta cuatro perfiles analíticos, útiles para encuadrar una discusión más específica. En primer lugar, se han de tener en cuenta las diferencias de desarrollo de la problemática bajo el perfil temporal en lo referente a los tres sectores ya citados de la legislación electoral en la normativa de la democracia pluralista; en segundo lugar, el diverso papel constitucional de la misma bajo el punto de vista material; en tercera instancia, el perfil técnico referente al sistema electoral en sentido estricto; $y$, en fin, la vinculación empíricamente evidenciada en las opciones realizadas entre los varios planos de la normativa en cuestión.

4.1. Desde el punto de vista temporal, el tema de la capacidad electoral activa y pasiva ha precedido a todos los demás, conectándose con la llamada forma de Estado y el régimen; en cambio, el sistema electoral en sentido estricto ha sido un argumento polémico en el tiempo de la crisis de participación; y en el siguiente a ésta se relacionó con la cuestión de la forma de gobierno; $y$ en fin, la legislación electoral de contorno constituye -en normativas con sistemas de partidos estabilizados- el tema más candente para la conexión con el significado cualitativo del acto electoral.

4.2. Desde un punto de vista más estrictamente constitucional, el sistema electoral en sentido estricto -sea la que fuere su cobertura 
en el ámbito del sistema de las fuentes- a nivel nacional constituye un conjunto normativo tendencialmente rígido, representando -como se ha dicho- una auténtica y verdadera norma de régimen, que conecta con la naturaleza y la dinámica de sujetos políticamente relevantes del sistema partítico.

4.3. Desde el punto de vista técnico, en lo que concierne al sistema electoral en sentido estricto, es posible elaborar instrumentos electorales selectivos incluso a través de la utilización de fórmulas proporcionales utilizando, por un lado, todas las potencialidades del mecanismo de transformación de los votos en escaños (tipo de selección, tipo y dimensión de los colegios, tipo de fórmula); y por otro lado, la conexión con las reglas de posición previa y funcionamiento de los órganos de la forma de gobierno, capaces de incidir en el sentido del debate con función agregadora.

4.4. Desde el punto de vista concreto, en la experiencia comparativa de las normas de la democracia pluralista normalmente hay coherencia en la normativa electoral aplicada a los niveles varios (nacional o federal, regional o estatal, local) y estabilidad sustancial de la misma en el tiempo. Esto es posible individuarlo no sólo en lo referente a los sectores relacionados con la forma de Estado (capacidad electoral, activa y pasiva; certeza de los procedimientos y legislación electoral de contorno), sino también en el sistema electoral en sentido estricto.

\section{EL CASO ITALIANO Y SU PECULARIEDAD}

En estas breves notas resulta notoria la peculariedad del caso italiano del último decenio, que se ha visto agredido por una crisis realmente de régimen (entendiendo con estos términos las normas, valores, reglas de juego y estructuras de autoridad en que actúan los sujetos políticamente relevantes), con la reestructuración-realineamiento todavía incompleto del sistema de partidos nacional $\vee$ local.

Recuerdo a este propósito que para comprender la situación actual resulta oportuno hacer referencia a los dos ciclos que han caracterizado la historia constitucional de la normativa en materia electoral, es decir, el periodo 1946-1993, caracterizado por la convención proporcionalista, y el de 1993, donde la razón de la transformación de los votos en escaños ha ido diferenciándose entre los distintos niveles representativos. 


\subsection{La convención proporcionalista y su dinámica de 1946 a 1993:}

a) La fase anterior a 1993 estuvo caracterizada por la imposibilidad de pensar en otros mecanismos de transformación de votos en escaños, que no estuvieran basados en la fórmula proporcional con resultado tendencialmente de calco en conexión con la forma de gobierno parlamentario. La peculiar índole de las fuerzas políticas y el fracaso del premio de mayoría hicieron que tras 1953, se desarrollara y profundizara la convención proporcionalista en todos los niveles.

b) En tal contexto la polémica sobre el sistema electoral quedó confinada muchos años al nivel científico-polémico; resurgió en los años 80 , en relación con la discusión sobre las innovaciones constitucionales; y hacia el final del decenio en cuestión, se convirtió en subversiva respecto del estado de las fuerzas que actuaban en el marco de la estrategia referendaria.

5.2. La crisis del régimen y las nuevas reglas electorales de 1993. En 1989 la crisis del sistema político italiano asumió caracteres cada vez más incisivos hasta transformarse en una auténtica crisis de régimen, derivada de factores de tipo internacional e interno.

a) El referendum sobre la preferencia única y los otros sobre el sistema electoral del Senado certificaron la disolución contradictoria de dos pilares de la organización republicana basada en los partidos y la crisis profunda de realineación del sistema. La clase política parlamentaria, asediada por "tangentópolis" y por problemas de autoconservación, consiguió renovar eficazmente sólo en lo referente al nivel de ayuntamientos con la Ley núm. 81/1993, pero fracasó en la parte de las leyes 276-277/1993, sobre el sistema electoral, a las que debe añadirse la ley 515/1993, referente a la legislación electoral de contorno.

La ley de 1995 sobre las regiones refleja una débil tendencia a aplicar el modelo neoparlamentario y en su experiencia práctica encuentra una serie de defectos que son tomados en consideración en el iter parlamentario de la ley constitucional 2/1999.

5.3. El efecto actual de tales hechos es que en la normativa en vigor coexisten diferentes sistemas electorales con un sistema de partidos en realineación incompleta.

En efecto, para los niveles europeo, nacional, regional, provincial y municipal están previstos sistemas electorales diferentes que complican todavía más la crisis del partido político desarrollada con el pasar de los años. Con el tiempo se ha pasado de la prevalencia de formacio- 
nes organizativas de masa durante los años cincuenta, a las que eran mezcla de todo en los dos decenios siguientes, para llegar luego a una configuración gelatinosa caracterizada por partidos en forma de "cartel» con fuertes elementos personales.

\section{PRECEDENTES E ITER DE LA INNOVACIÓN CONSTITUCIONAL DE 1999}

El debate sobre la modificación del sistema electoral regional tuvo un itinerario periestáltico en los veinte años últimos y con frecuencia asumió carácter de provisionalidad dentro del debate sobre la innovación institucional en Italia. La función esencialmente comunicativa de las propuestas ha hecho que se pasara indiferentemente del nivel nacional al local y luego al regional. Según se ha observado recientemente (Mariucci, en "Le istituzioni del federalismo", 1999, núm. 6, pp. 1149 ss.), desde mediados de los años ochenta ya se intentó diferenciar y estabilizar la normativa regional sobre la base de propuestas tendentes a modificar el mecanismo de transformación de votos en escaños, fundado en la fórmula proporcional, el tipo de la forma de gobierno asambleario adoptado en las regiones de estatuto ordinario.

Al final de los años ochenta, la investigación cognoscitiva de la comisión parlamentaria para las cuestiones regionales había visto que los expertos contemplaban varios tipos de soluciones (entre éstas, las neoparlamentarias y las presidenciales), mientras Massimo S. Giannini había presentado la hipótesis de los paquetes de formas de gobierno y sistemas electorales, entre los que las regiones deberían elegir la opción propia.

Todavía no era el momento de diferenciar. Por otro lado, la misma Comisión De Mita-lotti preveía que el art. 122 de la Constitución quedase idéntico, con el solo añadido de un último punto que daba a los Estatutos regionales la posibilidad de elegir una forma de gobierno diferente. En realidad, fue la Conferencia de los presidentes de las regiones que, entre 1993 y 1994, pidió la revisión del sistema electoral regional, dando así lugar a la solución in progress del llamado Tatarellum.

En el período siguiente, la iniciativa a este propósito por parte de la Conferencia de presidentes de las regiones y de la misma Anci, se sobrepuso al intento de la Comisión bicameral instituida con la ley Constitucional. 1/1997. Tras fracasar este intento con la hibernación de 
la Comisión, se reanudó la presión hasta llegar a las elecciones regionales de 2000 con una revisión constitucional de algunos elementos esenciales al respecto y una normativa transitoria de lo referente a la elección directa. La innovación constitucional del año pasado, caracterizada por un funcionamiento ondulatorio del proyecto en el ámbito de la dialéctica parlamentaria, nace en un contexto en el que a las incertidumbres del ámbito nacional corresponden diferenciaciones entre los varios niveles y dinámicas contradictorias bajo el perfil de la reestructuración institucional.

\section{LA INNOVACIÓN CONSTITUCIONAL DEL ART. 122}

Dentro de este marco teórico y práctico, la ley constitucional del 22 de noviembre 1999, en lo referente al sector de la legislación electoral incide en los casos:

- de inelegilibidad e incompatibilidad del presidente, de los otros miembros de la Junta regional y de los consejeros regionales;

- y en el mecanismo de transformación de los votos en escaños (o sistema de elección de los mismos).

Aunque pueda resultar innecesario recordarlo, resultan excluidos de la intervención autónoma de las regiones los sectores que se vinculan directamente a la forma de Estado, es decir, la capacidad electoral activa y también la pasiva (para esta última,dentro de los límites que se han de establecer) y la misma legislación electoral de contorno.

En lo referente al sistema electoral en sentido estricto concerniente al nombramiento del Presidente de la Junta y de los miembros del Consejo regional, y también las partes tocantes a la relación entre Junta y Consejo, el art. 2 de la ley const. en cuestión, parece dejar un margen notable de juego a las regiones; pero después las vincula a los límites de los principios fundamentales establecidos con la ley de la República, que establece asimismo la duración de los órganos electivos.

De modo más sustancial, la misma libertad de movimientos de las regiones en materia de forma de gobierno resulta limitada a la parlamentaria, si bien con la posibilidad de articularla en el ámbito de un continuum entre la clásica y la neoparlamentaria. De hecho quedan excluidas las hipótesis presidenciales y directoriales, a la vez que 
resulta evidente una potencial contradicción de sistema con la misma normativa transitoria, cuando según el art. 2 se dispone que «el Presidente de la Junta regional sea elegido con sufragio universal y directo, salvo que el estatuto regional disponga en contrarion.

En el marco de la laboriosa discusión parlamentaria, varias intervenciones recalcaron la necesidad de coherencia entre el módulo representado por el sistema electoral en sentido estricto y el ámbito de la forma de gobierno neoparlamentario, de modo que garantice el funcionamiento completo del modelo. Concretamente, en el debate en el Senado, el relator Fisichella (pp. 165 y ss.) pidió la aprobación de una normativa sobre los principios en cuestión, encaminada a evitar deformaciones en cuanto a la instalación del gobierno de legislatura. Trayendo el agua a sus tesis tradicionales, con esta perspectiva Fisichella parecía inclinarse a la adopción del sistema electoral mayoritario de doble turno en colegio uninominal. Sin cometer errores de tipo conceptual, Fisichella pedía demasiado porque, desde el punto de vista técnico, no es indispensable la coherencia entre fórmula mayoritaria en la elección de la presidencia y mecanismo de elección del Consejo.

Las opciones adoptadas por la normativa transitoria (v. art. 5 de la ley constitucional núm. 1, de 1999), que ciertamente tendrá un peso en el futuro institucional de las regiones, fueron fruto de intenso debate; y con la alternativa sobre el modo de elegir al Presidente de la Junta a base de un turno único o con segunda vuelta, están justificadas por intereses $y$ análisis de la realidad política e institucional muy diferentes. No obstante, el modelo preferencial descrito en el nuevo art. 122 Const., de características sustancialmente abiertas, podría no tenerse en cuenta al adoptar la subordinada que excluye la elección directa del Presidente de la Junta o por la adopción de mecanismos reflejos para la elección del Consejo. Con esta visual, las posibles opciones sobre las inelegibilidades e incompatibilidades serán ciertamente menos impactantes en el funcionamiento del conjunto institucional, en relación con las referentes al compartimento del sistema electoral en sentido estricto para la institución de los dos poderes de la estructura regional.

Por esto precisamente, en la ley sobre principios debería estar previsto un modelo general, es decir, un conjunto coherente de modelos -como sugería al final de los ochenta Massimo S. Giannini- por cuyo medio, más allá de la opción sobre la elección directa del Presidente de la Junta, se garantice la posibilidad de hallar una mayoría de entrada y sancionar con la disolución la posible inestabilidad de salida. En esta espera, el legislador debería tener la valentía de ampliar el 
ámbito tradicional del campo electoral hasta la fase de selección de las candidaturas reconociendo que, análogamente a cuanto decidió la Corte suprema estadounidense a mediados de los años cuarenta, la opción electoral puede resultar ya fijada en el momento de la presentación de las candidaturas oficiales.

\section{CONCLUSIONES. POSIBLES ALTERNATIVAS Y PELIGROS DE LA SITUACIÓN CONFUSA}

Cuando se redactan estas líneas está a punto de comenzar la actividad de los nuevos consejos regionales y se volverán a discutir las reglas estatutarias. No será un tiempo fácil, pero asumirá aspectos de interés institucional agudo.

Desde el punto de vista que nos interesa en esta sede, el problema esencial es la ley sobre los principios y los conflictos que podrían surgir de dicha ley. Según es sabido, los planteamientos sobre el tema son sustancialmente dos. El primero sostiene la necesidad de que la aprobación de la ley sobre los principios preceda al momento de la elección autónoma de las regiones, a fin de evitar centrifugaciones $y$ conflictos. En cambio, el segundo propone pensarlo en el periodo siguiente $o$, incluso, ni siquiera pensar en ello.

La última alternativa se basa en el hecho de que, en realidad, la ley sobre los principios no sería indispensable en el funcionamiento del sistema, ya que aquéllos podrían recuperarse dentro de la normativa vigente. Por otra parte, hay quien sostiene -según un perfil pragmáticoque la aprobación de dicha ley podría ser demasiado costosa para la mayoría de gobierno y no útil para la oposición actual en un momento en que no hay claridad sobre las reglas electorales a nivel nacional.

Una vez más, el problema del ajuste institucional de las regiones aparece, por tanto, necesariamente conectado con el nacional, por tres motivos al menos.

En primer lugar, porque la fase reconstructiva de las regiones está inserta en la fase de una larga campaña electoral, que se puede prever durará un año solar.

En segundo lugar, porque muchos de los eventos de la fase siguiente están conectados con el resultado del referendum electoral, que definirá las reglas para la elección de las Asambleas representativas en sentido actual y potencial. 
En tercer lugar, porque la ley sobre los principios puede ser funcional, no sólo según un modelo de rearticulación autónoma de la relación centro-periferia, sino según los intereses de los sujetos en reestructuración; éstos podrán puntualizarse claramente sólo cuando las nuevas cartas se distribuyan el año próximo.

Fundamentalmente se prepara una fase más fluida todavía de cuanto pueda pensarse, en la que las condiciones políticas concretas serán las que condicionen la selección de los temas en cuestión, como ya ocurrió en la fase constituyente de mayo-junio 1947. Si se contempla bajo esta luz específica, entonces todo el proceso de reestructuración regional será muy fluido, y planteará problemas también sobre lo referente a los sujetos políticamente relevantes dentro del juego sistémico y sus relaciones.

Ciertamente subsiste el interrogante sobre la coherencia, más general, de un proceso de innovación institucional que avanza sobre la base de la desestructuración del modelo preexistente, que funcionaba de manera netamente insuficiente respecto a los standars de las democracias industriales avanzadas; pero era coherente con la índole de la ordenación y construcción fatigosa de una pluralidad de realidades institucionales diversificadas, dentro de las cuales la nacional sigue sujeta a defectos tradicionales.

Por tanto, la atención al nivel regional esconde el problema más amplio de la innovación institucional en Italia y la necesidad persistente y principal de modificar las reglas a nivel nacional. Para tal objetivo, en mi opinión, el primero y más importante paso no es tanto ni es sólo la adopción de mecanismos mayoritarios respecto del sistema electoral en sentido estricto, sino la modificación del bicameralismo perfecto que caracteriza al modelo de forma de gobierno delineado por la Constitución de 1948 y la transformación del Senado en Cámara representativa de las instituciones regionales. El mismo referendum del 21 de mayo (si consigue obtener un resultado objetivol, quedará difuminado por la existencia de la actual ordenación institucional que -como ha sucedido dos veces con el sistema regulado por las leyes 276 y 277- comportará la elección de las dos Cámaras con mayoría no homogénea. Se trata de una realidad que no se encuentra en ninguna otra ordenación de democracia pluralista, que confía la estabilidad gubernamental a la probabilidad de acertar el 5 más uno en la lotería.

Las conclusiones de mi intervención son, por tanto, muy abiertas y llenas de preocupación; pero unen el plano nacional al regional con vinculación indestructible que sólo posturas irrealistas pueden olvidar. 